\title{
Cardiopulmonary exercise testing is a better outcome predictor than exercise echocardiography in asymptomatic aortic stenosis
}

\author{
Olivia Domanski ${ }^{a, *, 1}$, Marjorie Richardson ${ }^{\mathrm{a}, 1}$, Augustin Coisne ${ }^{\mathrm{a}, \mathrm{b}, \mathrm{c}, \mathrm{d}, \mathrm{e}}$, Anne-Sophie Polge ${ }^{\mathrm{a}}$, Stephanie Mouton a , \\ François Godart ${ }^{\mathrm{a}, \mathrm{b}}$, Jean Louis Edmé ${ }^{\mathrm{a}, \mathrm{b}}$, Regis Matran ${ }^{\mathrm{a}, \mathrm{b}, \mathrm{d}}$, Patrizio Lancellotti ${ }^{\mathrm{f}, \mathrm{g}}$, David Montaigne ${ }^{\mathrm{a}, \mathrm{b}, \mathrm{c}, \mathrm{d}, \mathrm{e}, *}$ \\ a CHU Lille, Department of Echocardiography and Physiology, Department of Pediatric Cardiology and Congenital Heart Diseases, F-59000 Lille, France \\ b Univ. Lille, F-59000 Lille, France \\ c Inserm UMR 1011, F-59000 Lille, France \\ ' Institut Pasteur de Lille, F-59000 Lille, France \\ e European Genomic Institute for Diabetes (E.G.I.D.), FR 3508, F-59000 Lille, France \\ ${ }^{\mathrm{f}}$ University of Liège Hospital, GIGA Cardiovascular Sciences, Departments of Cardiology, Heart Valve Clinic, CHU Sart Tilman, Liège, Belgium \\ ${ }^{g}$ Gruppo Villa Maria Care and Research, Anthea Hospital, Bari, Italy
}

\section{A R T I C L E I N F O}

Article history:

Received 30 May 2016

Received in revised form 25 October 2016

Accepted 27 October 2016

Available online $\mathrm{xxxx}$

\section{Keywords:}

Aortic stenosis

Cardiopulmonary exercise testing

Exercise echocardiography

Peak oxygen consumption

Aerobic capacity

\begin{abstract}
A B S T R A C T
Background: Objective assessment of maximal aerobic capacity using peak oxygen consumption (peak VO2) can be helpful in the management of patients with asymptomatic aortic stenosis (AS). The relationship between peak VO2 and AS severity criteria derived from rest and supine exercise echocardiography (SEE) has never been explored.

Objectives: We aimed to determine whether low peak VO2 ( $<85 \%$ of predicted value) is associated with severity parameters in SEE, and poor clinical outcome.

Methods: Fifty one asymptomatic patients (mean age of $54 \pm 21$ years) with moderate to severe aortic stenosis $(\operatorname{Vmax}>3 \mathrm{~m} / \mathrm{s})$ and left ventricle ejection fraction $>50 \%$ prospectively underwent resting and SEE and cardiopulmonary exercise testing (CPX).

Results: Peak VO2 was lower than expected $(21.9 \pm 7.4 \mathrm{~mL} / \mathrm{kg} / \mathrm{min})$, i.e. $<85 \%$ of predicted value in $57 \%$ patients, secondary to cardiac limitation in most of them (69\%). In multiple regression analysis, age, BMI and female gender were the only independent determinants of peak VO2. Interestingly no parameter derived from SEE was associated with peak VO2. After $21 \pm 7$ month follow-up, no patient died, 20 underwent cardiac surgery. Peak VO2 $<85 \%$ of predicted value was associated with lower event free survival compared to normal peak VO2 $(57 \% \pm 11 \%$ vs $93 \pm 6 \%, \mathrm{p}=0.036)$ whereas no exercise echocardiographic parameter could predict such events. Peak VO2 $\geq 85 \%$ had a negative predictive value of $97 \%$.

Conclusion: $\mathrm{CPX}$ detects a high proportion of false asymptomatic AS patients with poorer outcome that cannot be predicted by SEE markers of AS severity. Assessment of aerobic capacity should be part of current approach within a "watchful waiting" strategy.
\end{abstract}

(C) 2016 Elsevier Ireland Ltd. All rights reserved.

\section{Introduction}

Severe aortic stenosis (AS) is the most common indication for valve replacement in United States and Europe with aging of population. Current guidelines recommend aortic valve replacement for severe AS in case of symptoms or when left ventricle ejection fraction (LVEF) is

Abbreviations: AS, Aortic stenosis; BMI, Body Mass Index; CPX, Cardiopulmonary exercise testing; EPHT, Exercise pulmonary hypertension; LVEF, Left ventricle ejection fraction; MGI, Mean gradient increase; PHT, Pulmonary hypertension; TAPSE, Tricuspid annular plane systolic excursion; VO2, Oxygen consumption.

* Corresponding authors at: Service d'Explorations Fonctionnelles Cardiovasculaires, CHU Lille, 59037 Lille cedex, France.

E-mail address: Olivia.domanski@chru-lille.fr (O. Domanski).

${ }^{1}$ O.D. and M.R. equally contributed to this work.
$<50 \%$ [1] as surgery improves symptoms, LV function and survival. Concerns remain regarding the best therapeutic option for asymptomatic patients with moderate to severe AS and preserved LVEF. On the one hand, sudden cardiac death not preceded by symptoms occurs in $1 \%$ of these patients per year, and early surgery has recently been suggested in centers with very low operative risk [2-6]. On the other hand, isolated aortic valve replacement carries on operative mortality of 2.8 to $3.7 \%$ in large recent registries [1,7], and referring too early asymptomatic patients to premature surgery exposes them to the potential risk for long-term valve prosthesis complications.

As elective surgery cannot be recommended in every patient with asymptomatic severe aortic stenosis, reliable risk estimation, appropriate AVR indication and timing are important clinical challenges. In this approach, the accurate determination of symptoms is crucial 
and currently based on systematic exercise testing to unmask symptoms or abnormal blood pressure response. Indeed many patients may unconsciously reduce their level of physical activity [1].

In the past few years, both supine exercise echocardiography and cardiopulmonary exercise testing (CPX) have been proposed for risk stratification in asymptomatic AS. Decrease of LVEF ( $>3 \%$ ), significant elevation in transaortic mean gradient $(>18 \mathrm{~mm} \mathrm{Hg}$ ) or pulmonary hypertension (PHT) (>60 mm Hg) during supine exercise echocardiography has been associated with cardiac events, i.e. aortic valve replacement or cardiovascular death [8-11]. Peak oxygen consumption (peak VO2) measured during CPX was found to objectively assess functional capacity in this population and may predict survival in retrospective studies $[12,13]$.

No study has prospectively explored the additive prognosis value of CPX on top of current prognosis markers yet. We hypothesized that alteration of aerobic capacity (defined as peak VO2 $<85 \%$ of predicted value) in asymptomatic AS could be associated with severity parameters in supine exercise echocardiography, and with poor clinical outcome.

\section{Methods}

\subsection{Study population}

Consecutive asymptomatic patients with moderate to severe AS (peak aortic jet velocity $\geq 3 \mathrm{~m} / \mathrm{s}$ and preserved LVEF $>$ 50\%) referred to our center from December 2012 to June 2015 for risk stratification underwent resting and supine exercise echocardiography, CPX and biology. The referring cardiologist recorded medical history and carefully checked the absence of any symptoms. Exclusion criteria were: (i) symptoms including dyspnea, angina or syncope, (ii) more than mild aortic or mitral regurgitation at resting echocardiography, (iii) history of aortic valve surgery, (iv) atrial fibrillation, (v) LVEF $<50 \%$, and (vi) inability to perform supine exercise echocardiography or CPX All patients gave informed consent for these explorations during the first medical visit to our hospital in accordance with local ethical committee.

\subsection{Resting and supine exercise echocardiography}

Echocardiographic examination was performed using a Vivid E9 Ultrasound system (General Electrics Healthcare). All Doppler echocardiographic data were stored on a dedicated workstation (Echo-PAC software, General Electrics Healthcare) for subsequent offline analysis.

Before exercise test, all patients underwent a comprehensive resting examination that included M-mode, two-dimensional echocardiography, pulsed-wave and continuous-wave Doppler measurements, according to guidelines [1,14-16], by an experienced echocardiographist. The assessment of systolic function was made by Simpson's biplane method at rest and peak exercise. Multiple transducer positions were used to record peak aortic jet velocities, and aortic valve area was calculated with the continuity equation [17], after careful measurement of left ventricular outflow tract (LVOT) diameter. Standard 2D greyscale images focused on the LV were acquired in 4-chamber, 2-chamber and long-axis views at high frame rates (between 50 and $80 / \mathrm{s}$ ) in order to obtain LV global longitudinal strain by 2D speckle tracking, using ECHO-PAC dedicated software (General Electrics Healthcare). If necessary the region of interest automatically tracked by the device was manually corrected. Pulmonary hypertension and valvulo-arterial impedance (Zva) were calculated as previously described $[10,18]$.

A symptom-limited maximal bicycle exercise test was performed the same day in the semi-supine position (eBike EL, General Electrics Healthcare). All exercise 2D and Doppler data were obtained at $25 \mathrm{~W}$ and at peak exercise as previously described by our group [19]. No medication was stopped before the examination. After an initial workload of $25 \mathrm{~W}$ maintained for $2 \mathrm{~min}$, it was increased every 2 min by $20 \mathrm{~W}$. A 12-lead ECG was monitored continuously and blood pressure was measured at rest and every 2 min during exercise. Aortic transvalvular maximal velocity was recorded in apical 5-chamber view during exercise. Abnormal test was defined as: (i) occurrence of angina, dizziness or syncope, (ii) complex ventricular arrhythmia, or (iii) fall of systolic blood pressure during exercise $\geq 20 \mathrm{~mm} \mathrm{Hg}$.

Exercise $\mathrm{PHT}>60 \mathrm{~mm} \mathrm{Hg}$, increase of mean transaortic gradient $>18 \mathrm{~mm} \mathrm{Hg}$ and decrease of $\mathrm{LVEF}>3 \%$ were considered as exercise echocardiographic parameters of bad prognosis as previously described [9-11].

\subsection{Cardiopulmonary exercise testing (CPX)}

All patients underwent a treadmill CPX on an Ergoline $800 ®$ bicycle (Sensormedics, Yorba Linda, United States) with respiratory gas-exchange analysis, using a modified Bruce protocol (progressive increase in workload of $10-15 \mathrm{~W} / \mathrm{min}$ ) as already described by our group [20]. Each CPX was performed within the month following echocardiographic examination. Patients' medications were not stopped for the examination. Patients were encouraged to exercise until exhaustion. Blood pressure was measured every 2 min; continuous 12-lead electrocardiogram and saturation monitoring (SpO2) were recorded. Breath-by-breath minute ventilation (VE), carbon dioxide production (VCO2), their ratio (VE/VCO2), and oxygen consumption (VO2) were measured using a pneumotach (Oxycon Pro, Viasys, France). Room temperature, barometric and hygrometric pressure were noted. A calibration of the pneumotach was realized before every exam using a dosing syringe of $2 \mathrm{~L}$, first slowly then quickly. The respiratory exchange ratio (RER) was defined as VCO2/VO2. Peak VO2 was expressed as absolute peak or normalized peak (percent of predicted value derived from Wasserman formula) [21]. The VE/VCO2 slope was calculated by linear regression. The 02 -pulse, an estimate of stroke volume, was calculated as $\mathrm{VO} 2 /$ heart rate. Altered 02 -pulse was defined as $<85 \%$ of predicted value or $<100 \%$ when patients were under beta blocker therapy (due to induced bradycardia under treatment). LV stroke volume was calculated at peak exercise using Wasserman's equation [21]. Abnormal test was defined with the same criteria that exercise echocardiography. Exercise was considered maximal when patients reached $85 \%$ of predicted maximum heart rate, or when RER was $\geq 1.15$. Functional capacity was considered reduced when peak VO2 was $<85 \%$ of predicted value. Cardiac limitation was defined as a peak VO2 $<85 \%$ associated with a low 02 -pulse as previously described [22]. Pulmonary limitation was defined as peak $\mathrm{VO} 2<85 \%$ associated with ventilatory reserve $<20 \%$ or occurrence of oxygen blood desaturation (SpO2 $<92 \%$ ) during exercise. Muscular limitation was diagnosed when functional capacity was reduced without cardiac or pulmonary limitation. Venous blood sample was taken just before CPX for hemoglobin and NTproBNP measurements.

\subsection{Patients' follow-up}

Patients were prospectively followed after the initial examination every 6 months. Particular care was taken to obtain information for symptom onset, surgical aortic valve replacement and death. The clinical management was determined independently by the patient's personal physician.

Events were defined as occurrence of cardiovascular death or need for aortic valve replacement motivated by the development of symptoms or LV systolic dysfunction $(\mathrm{LVEF}<50 \%)$.

\subsection{Statistical analysis}

Continuous variables with a Gaussian distribution are given as mean \pm SD. Continuous variables with no Gaussian distribution are given as median [25th to 75th] percentiles. Categorical variables are given as the number (percentage) of patients with the respective attribute. Bivariate comparisons were performed using the t-test for normally distributed continuous variables or the Mann-Whitney $\mathrm{U}$ test for variables not normally distributed. Bivariate comparisons of categorical variables were performed with the chi-square test. Pearson's test was performed to evaluate potential correlations between peak VO2 and clinical, biological and echocardiographic parameters. Potential predictors for peak VO2 with p-value $<0.05$ on bivariate analysis were entered in a multivariate stepwise linear regression model using backward variable selection. Exercise echocardiographic severity criteria were forced into the model (exercise PHT $>60 \mathrm{~mm} \mathrm{Hg}$, mean aortic gradient increase $>18 \mathrm{~mm} \mathrm{Hg}$, LV ejection fraction decrease $>3 \%$ ) because of their clinical relevance. Event rates were estimated using Kaplan-Meier method and compared using a $\log$-rank test. A value of $\mathrm{p}<0.05$ was considered statistically significant. All analyses were conducted using Medcalc Software Version 15.8 (MedCalc Software bvba, Ostend, Belgium). Figures were drawn with GraphPad Prism v5.0.

\section{Results}

\subsection{Patient characteristics and supine exercise echocardiography}

Patient characteristics are presented in Table 1. Fifty one patients with asymptomatic aortic stenosis (LV aortic valve area $0.97 \pm$ $0.24 \mathrm{~cm}^{2}, \operatorname{Vmax} 4.04 \pm 0.74 \mathrm{~m} / \mathrm{s}$ ) and preserved ejection fraction (LVEF $\geq 50 \%$ ) were explored. Aortic stenosis was secondary to a bicuspid valve in 35 patients (69\%), degenerative in 12 patients, rheumatic in 2 patients and due to congenital valve hypoplasia in 2 patients. Our population was relatively young ( $54 \pm 21$ years) with a sex ratio of nearly 1 and few co-morbidities. Most of the patients had normal blood NT-proBNP level.

Supine exercise was performed to exhaustion in all patients without any complex ventricular arrhythmia or criteria for myocardial ischemia. Almost half of the patients displayed exercise echocardiographic criteria of poor prognosis (Table 2): 4 patients ( $8 \%$ ) developed exercise PHT, $10(20 \%)$ presented an abnormal increase of mean trans-aortic gradient (>18 $\mathrm{mm} \mathrm{Hg}$ ) and 10 (20\%) revealed a decrease of LVEF at peak exercise. 


\subsection{Aerobic capacity in asymptomatic aortic stenosis}

Peak VO2 was unexpectedly low for these asymptomatic middleaged patients (i.e. $21.9 \pm 7.4 \mathrm{~mL} / \mathrm{kg} / \mathrm{min}$ or $83 \pm 17 \%$ of predicted value). Twenty nine patients (57\%) had reduced aerobic capacity defined as peak VO2 $<85 \%$ of predicted value (Table 3 ). Most of these patients ( $n=20,69 \%)$ displayed criteria for cardiac limitation with (i) normal hemoglobin but low 02 -pulse at peak exercise and (ii) accordingly lower calculated LV stroke volume at peak exercise than patients with peak VO2 $\geq 85 \%$ of predicted value ( $60 \pm 25$ vs $85 \pm 28 \mathrm{~mL}, \mathrm{p}=0.01$ ).

Of note, an abnormal decrease in systolic blood pressure was observed in 3 patients, among which two had peak VO2 < 85\% of predicted value.

Compared to patients with conserved aerobic capacity, patients with decreased peak VO2 $(<85 \%$ of predicted value) were more often overweight and diabetic, with higher heart rate and transtricuspid pressure gradient at rest. AS severity did not differ between the 2 groups (aortic valve area: $0.98 \pm 0.24$ vs $0.96 \pm 0.25 \mathrm{~cm}^{2}, \mathrm{p}=0.71$; peak aortic jet velocity: $4.09 \pm 0.81$ vs $3.99 \pm 0.66 \mathrm{~m} / \mathrm{s}, \mathrm{p}=0.66$ ). Surprisingly, there was no significant difference between the two groups regarding echocardiographic data at supine exercise, even for maximal workload ( $94 \pm 36$ vs $101 \pm 49 \mathrm{~W}, \mathrm{p}=0.57$ ). Exercise echocardiographic criteria of poor prognosis were equally present in the two groups.

To better understand poor aerobic capacity in patients with asymptomatic aortic stenosis, we further explored correlations of peak VO2 $(\mathrm{mL} / \mathrm{min} / \mathrm{kg})$. In bivariate analysis, peak VO2 significantly correlated with numerous parameters, i.e. age, gender, weight status, history of diabetes mellitus and hypertension, bicuspid valve, $\mathrm{E} / \mathrm{e}^{\prime}$ ratio at rest and peak exercise, LV aortic valve area at rest and peak exercise, transtricuspid pressure gradient (Tables 4 and 5).

Yet, after multivariate adjustment, the only independent determinants of peak VO2 were age, BMI and female gender (Table 6). Aortic stenosis severity and exercise echocardiographic criteria of poor prognosis (i.e. mean aortic valve gradient increase $>18 \mathrm{~mm} \mathrm{Hg}$, LVEF decrease $>3 \%$, or exercise PHT $>60 \mathrm{~mm} \mathrm{Hg}$ ) were not independently associated with peak VO2 (Fig. 1; Table 5).

\subsection{Predictors of outcome in asymptomatic aortic stenosis}

Among our population of 51 patients, no patient died during the $21 \pm 7$ month follow-up, 20 underwent aortic valve surgery. As expected, no event occurred before 9 months of follow-up since enrolled patients were asymptomatic patients with normal LVEF.

Five patients underwent surgery for other reasons than symptoms or onset of LV dysfunction: 1 for pregnancy desire, 1 for endocarditis, 3 for young age (the presence of symptoms being hard to evaluate under 20 years old) and were therefore excluded from survival statistics.

There was no difference in survival between patients having exercise echocardiographic parameters of severity (i.e. exercise PHT $>60 \mathrm{~mm} \mathrm{Hg}$, transaortic gradient increase $>18 \mathrm{~mm} \mathrm{Hg}$ and/or LVEF decrease $>3 \%$ ) and the others (free event survival, $80 \pm 11 \%$ versus $70 \pm 11 \%$ respectively, $\mathrm{p}=0.38$ ) (Fig. $2 \mathrm{~A}$ ). Conversely, patients with conserved aerobic capacity (peak VO2 $\geq 85 \%$ of predicted value) had a higher rate of event-free survival at $21 \pm 7$ months follow-up ( $93 \pm 6 \%$ vs $57 \pm 11 \%$, p $=0.036$ ) (Fig. 2 B). Peak VO2 was thus a better outcome predictor than exercise echocardiographic parameters of severity in our asymptomatic AS patients. Peak VO2 $\geq 85 \%$ had a negative predictive value of $97 \%$ to develop symptoms or $\mathrm{LV}$ dysfunction.

\section{Discussion}

We report here the first prospective work comparing semi-supine exercise echocardiography with cardio-pulmonary exercise test for the close follow-up of a moderate to severe asymptomatic aortic
Table 1

Clinical, biological, echocardiographic data at rest.

\begin{tabular}{|c|c|c|c|c|}
\hline & Overall & $\begin{array}{l}\text { Peak } \\
\text { VO2 }<85 \% \\
\text { PV }\end{array}$ & $\begin{array}{l}\text { Peak } \\
\text { VO2 } \geq 85 \% \\
\text { PV }\end{array}$ & $\mathrm{p}$ \\
\hline & $\mathrm{n}=51$ & $\mathrm{n}=29$ & $\mathrm{n}=22$ & \\
\hline \multicolumn{5}{|l|}{ Clinical } \\
\hline Age (years) & $54 \pm 21$ & $51 \pm 21$ & $56 \pm 21$ & 0.42 \\
\hline Female gender, n (\%) & $24(47 \%)$ & $14(48 \%)$ & $10(45 \%)$ & 0.99 \\
\hline BMI $\left(\mathrm{kg} / \mathrm{m}^{2}\right)$ & $26 \pm 5$ & $28 \pm 5$ & $25 \pm 4$ & 0.03 \\
\hline Body surface area $\left(\mathrm{m}^{2}\right)$ & $1.78 \pm 0.21$ & $1,83 \pm 0,18$ & $1,71 \pm 0,24$ & 0.06 \\
\hline Betablocker & $14(27 \%)$ & $8(28 \%)$ & $6(28 \%)$ & 0.99 \\
\hline \multicolumn{5}{|l|}{ Risk factors } \\
\hline Smokers & $9(18 \%)$ & $6(21 \%)$ & $3(14 \%)$ & 0.71 \\
\hline Diabetes mellitus & $10(20 \%)$ & $10(34 \%)$ & $0(0 \%)$ & $<0.01$ \\
\hline Hypertension & $20(39 \%)$ & $13(45 \%)$ & $7(32 \%)$ & 0.99 \\
\hline Dyslipidemia & $14(27 \%)$ & $9(31 \%)$ & $5(23 \%)$ & 0.54 \\
\hline Known coronary disease & $2(4 \%)$ & $1(3 \%)$ & $1(5 \%)$ & 1 \\
\hline \multicolumn{5}{|l|}{ Biologic } \\
\hline Hemoglobin (g/dL) & $14.0 \pm 1.6$ & $14.3 \pm 1.9$ & $14.2 \pm 1.2$ & 0.77 \\
\hline NT-proBNP (ng/mL) & $253[62 ; 224]$ & $77[33 ; 163]$ & $113[83 ; 327]$ & 0.59 \\
\hline \multicolumn{5}{|l|}{ Echographic data at rest } \\
\hline Heart rate (beats/min) & $75 \pm 15$ & $79 \pm 15$ & $70 \pm 14$ & 0.02 \\
\hline $\begin{array}{l}\text { Systolic arterial } \\
\text { pressure }(\mathrm{mm} \mathrm{Hg})\end{array}$ & $140 \pm 19$ & $137 \pm 16$ & $143 \pm 21$ & 0.31 \\
\hline Bicuspid valve & $35(69 \%)$ & $19(66 \%)$ & $16(73 \%)$ & 0.76 \\
\hline Left atrial area $\left(\mathrm{cm}^{2}\right)$ & $21 \pm 5$ & $22 \pm 6$ & $20 \pm 4$ & 0.15 \\
\hline $\mathrm{E} / \mathrm{E}^{\prime}$ ratio & $10 \pm 6$ & $11 \pm 8$ & $8 \pm 4$ & 0.11 \\
\hline $\begin{array}{l}\text { LV end-diastolic } \\
\text { volume }(\mathrm{mL})\end{array}$ & $96 \pm 23$ & $96 \pm 22$ & $96 \pm 24$ & 0.95 \\
\hline LV mass index $\left(\mathrm{g} / \mathrm{m}^{2}\right)$ & $100 \pm 26$ & $106 \pm 32$ & $93 \pm 14$ & 0.27 \\
\hline LV ejection fraction (\%) & $66 \pm 6$ & $65 \pm 7$ & $67 \pm 4$ & 0.22 \\
\hline $\begin{array}{l}\text { Global longitudinal } \\
\text { strain (\%) }\end{array}$ & $-19.6 \pm 2.9$ & $-19.2 \pm 2.6$ & $-20.0 \pm 3.3$ & 0.36 \\
\hline LV aortic valve area $\left(\mathrm{cm}^{2}\right)$ & $0.97 \pm 0.24$ & $0.98 \pm 0.24$ & $0.96 \pm 0.25$ & 0.71 \\
\hline $\begin{array}{l}\text { LV aortic valve } \\
\quad \text { area }<0,06 \mathrm{~cm}^{2} / \mathrm{m}^{2}\end{array}$ & $34(67 \%)$ & $20(69 \%)$ & $14(63 \%)$ & 0.77 \\
\hline Aortic jet velocity (m/s) & $4.04 \pm 0.74$ & $4.09 \pm 0.81$ & $3.99 \pm 0.66$ & 0.66 \\
\hline Aortic jet velocity $>4 \mathrm{~m} / \mathrm{s}$ & 25 (49\%) & $16(55 \%)$ & $9(41 \%)$ & 0.40 \\
\hline Aortic jet velocity $>5 \mathrm{~m} / \mathrm{s}$ & $4(8 \%)$ & $4(14 \%)$ & $0(0 \%)$ & 0.12 \\
\hline $\begin{array}{l}\text { Mean transaortic pressure } \\
\text { gradient }\end{array}$ & $40 \pm 16$ & $41 \pm 16$ & $40 \pm 16$ & 0.87 \\
\hline LV stroke volume $(\mathrm{mL})$ & $84 \pm 16$ & $84 \pm 15$ & $84 \pm 17$ & 0.91 \\
\hline $\mathrm{ZVA}\left(\mathrm{mm} \mathrm{Hg} / \mathrm{mL} / \mathrm{m}^{2}\right)$ & $3.69 \pm 1.24$ & $3.67 \pm 1.31$ & $3.67 \pm 1.18$ & 0.41 \\
\hline $\begin{array}{l}\text { Transtricuspid pressure } \\
\text { gradient }(\mathrm{mm} \mathrm{Hg})\end{array}$ & $25 \pm 8$ & $27 \pm 9$ & $22 \pm 5$ & 0.03 \\
\hline TAPSE (mm) & $22 \pm 4$ & $22 \pm 5$ & $23 \pm 3$ & 0.8 \\
\hline
\end{tabular}

Data presented are number of patients (percent) or mean $\pm \mathrm{SD}$. p-Value $<0.05$ was considered significant. BMI, Body Mass Index; LV, left ventricle; PV, predicted value; TAPSE, tricuspid annular plane systolic excursion.

stenosis population. Our main findings are that (i) a significant proportion of asymptomatic AS patients have low peak VO2 due to cardiac origin, with low $\mathrm{O} 2$ pulse, (ii) peak VO2 was significantly lower in overweight patients, with history of diabetes, higher resting heart rate and transtricuspid gradient at rest, (iii) peak VO2 did not correlate either with resting parameters of AS severity, nor with exercise echocardiographic parameters of poor prognosis, and (iv) low peak VO2 predicted poor outcome in this population.

\subsection{CPX in asymptomatic AS patients}

In fact, CPX splits the asymptomatic AS population in two groups revealing false asymptomatic patients with low peak VO2 $(<85 \%$ of predicted value). Indeed, in our work, a significant proportion of asymptomatic patients (57\%) had a low peak VO2. Recently, Dhoble et al. also showed in a population of 151 moderate to severe AS patients that $54 \%$ of them had an altered peak VO2 [13]. Interestingly, we first 
Table 2

Echocardiographic data at exercise.

\begin{tabular}{|c|c|c|c|c|}
\hline & \multirow{2}{*}{$\frac{\text { Overall }}{\mathrm{n}=51}$} & \multirow{2}{*}{$\frac{\text { Peak VO2 }<85 \% \text { PV }}{\mathrm{n}=29}$} & \multirow{2}{*}{$\frac{\text { Peak VO2 } \geq 85 \% \text { PV }}{n=22}$} & \multirow[t]{2}{*}{$\mathrm{p}$} \\
\hline & & & & \\
\hline \multicolumn{5}{|l|}{ Echographic data at exercise } \\
\hline Systolic arterial pressure (mm Hg) & $138 \pm 27$ & $155 \pm 25$ & $165 \pm 24$ & 0.19 \\
\hline Maximal heart rate (beats/min) & $83 \pm 12$ & $142 \pm 28$ & $134 \pm 26$ & 0.3 \\
\hline Maximal heart rate (\% theory) & $83 \pm 12$ & $84 \pm 12$ & $83 \pm 12$ & 0.76 \\
\hline Peak workload (W) & $96 \pm 41$ & $94 \pm 36$ & $101 \pm 49$ & 0.57 \\
\hline $\mathrm{E} / \mathrm{E}^{\prime}$ ratio & $10 \pm 4$ & $10 \pm 5$ & $9 \pm \overline{3}$ & 0.56 \\
\hline LV ejection fraction (\%) & $70 \pm 7$ & $70 \pm 7$ & $71 \pm 6$ & 0.53 \\
\hline Global strain 25 W (\%) & $-21.3 \pm 2.4$ & $-21.6 \pm 2.7$ & $-21.2 \pm 2.3$ & 0.69 \\
\hline LV aortic valve area $\left(\mathrm{cm}^{2}\right)$ & $1.10 \pm 0.38$ & $1.12 \pm 0.35$ & $1.07 \pm 0.43$ & 0.66 \\
\hline Aortic jet velocity (m/s) & $4.38 \pm 0.76$ & $4.45 \pm 0.78$ & $4.3 \pm 0.78$ & 0.54 \\
\hline Mean transaortic pressure gradient & $49 \pm 17$ & $51 \pm 18$ & $47 \pm 15$ & 0.41 \\
\hline LV stroke volume $(\mathrm{mL})$ & $81 \pm 26$ & $76 \pm 29$ & $87 \pm 20$ & 0.15 \\
\hline Transtricuspid pressure gradient $(\mathrm{mm} \mathrm{Hg}$ ) & $41 \pm 11$ & $41 \pm 12$ & $42 \pm 10$ & 0.81 \\
\hline TAPSE $(\mathrm{mm})$ & $25 \pm 4$ & $25 \pm 3$ & $25 \pm 3$ & 0.55 \\
\hline \multicolumn{5}{|l|}{ Exercise criteria of poor prognosis: } \\
\hline LV ejection fraction decrease $>3 \%$ & $10(20 \%)$ & $5(17 \%)$ & $5(23 \%)$ & 0.73 \\
\hline Mean gradient increase $>18 \mathrm{~mm} \mathrm{Hg}$ & $10(20 \%)$ & $4(14 \%)$ & $6(27 \%)$ & 0.29 \\
\hline Exercise $\mathrm{PHT}>60 \mathrm{~mm} \mathrm{Hg}$ & $4(8 \%)$ & $1(3 \%)$ & $3(14 \%)$ & 0.30 \\
\hline
\end{tabular}

Data presented are number of patients (percent) or mean \pm SD. p-Value $<0.05$ was considered significant.

LV, left ventricle; PHT, pulmonary hypertension; PV, predicted value; TAPSE, tricuspid annular plane systolic excursion.

demonstrated that this alteration resulted mainly from cardiac limitation since $69 \%$ of patients had also a low oxygen pulse in the absence of anemia.

The only three independent determinants of poor aerobic capacity (peak VO2 $<85 \%$ of predicted value) in our study were age, female gender (as previously described) [23,24], and BMI. Peak VO2 is well known to depend on age, gender and weight [21].

No marker of AS severity or LV function obtained at rest was able to predict peak VO2 in our work. Accordingly, Dulgheru et al. did not find any significant correlation between peak VO2 and LVEF or parameters of AS severity among 44 older ( $66 \pm 13$ years) asymptomatic moderate to severe AS patients [23]. Interestingly, these authors showed that LV longitudinal function of the basal myocardial segments is an independent determinant of peak VO2, by contrast to global longitudinal strain.
We did not specifically explore basal longitudinal strain, but we showed as Dulgheru et al. that LVEF is probably not the best index to assess LV systolic performance in AS although it is the only LV function parameter used to recommend AVR in asymptomatic patients with severe AS.

In another study, Steadman et al. found that peak VO2 was not associated with late gadolinium enhancement, or myocardial performance reserve using cardiac magnetic resonance, among 46 severe AS patients. Again no link between echocardiographic measurements of AS severity and peak VO2 was established [25].

Interestingly, limitation in peripheral oxygen transport and utilization have been observed in patients with preserved and altered heart failure because of reduced capillary density in skeletal muscle, impaired sympatholysis, decreased mitochondrial biogenesis and selective loss of type 1 oxidative muscle fibers [26]. Besides, exaggerated ventilatory

Table 3

Cardiopulmonary exercise test data.

\begin{tabular}{|c|c|c|c|c|}
\hline & \multirow{2}{*}{$\begin{array}{l}\text { Overall } \\
\mathrm{n}=51\end{array}$} & \multirow{2}{*}{$\frac{\text { Peak VO2 }<85 \% \text { PV }}{n=29}$} & \multirow{2}{*}{$\frac{\text { Peak VO2 } \geq 85 \% \text { PV }}{n=22}$} & \multirow[t]{2}{*}{$\mathrm{p}$} \\
\hline & & & & \\
\hline \multicolumn{5}{|l|}{ Cardiopulmonary exercise test data } \\
\hline VEMS (L) & $2.64 \pm 0.79$ & $2.60 \pm 0.82$ & $2.69 \pm 0.79$ & 0.39 \\
\hline VEMS/CVF $<70 \%$ & $1(2 \%)$ & $1(3 \%)$ & $0(0 \%)$ & 1 \\
\hline RER & $1.16 \pm 0.09$ & $1.18 \pm 0.1$ & $1.14 \pm 0.07$ & 0.28 \\
\hline Systolic arterial pressure (mm Hg) & $167 \pm 24$ & $171 \pm 25$ & $161 \pm 23$ & 0.25 \\
\hline Drop of systolic arterial pressure, n (\%) & $3(6 \%)$ & $1(3 \%)$ & $2(9 \%)$ & 0.57 \\
\hline Maximal heart rate (beats/min) & $146 \pm 31$ & $150 \pm 33$ & $164 \pm 22$ & 0.39 \\
\hline Maximal heart rate (\% PV) & $87 \pm 12$ & $88 \pm 14$ & $86 \pm 9$ & 0.58 \\
\hline Peak workload (W) & $114 \pm 47$ & $106 \pm 40$ & $124 \pm 54$ & 0.18 \\
\hline Peak VO2 (mL/kg/min) & $21.9 \pm 7.4$ & $19 \pm 6.1$ & $25.7 \pm 7.3$ & $<0.01$ \\
\hline Peak VO2 (\% PV) & $83 \pm 17$ & $71 \pm 8$ & $99 \pm 13$ & $<0.01$ \\
\hline Maximal $\mathrm{O} 2$ pulse $(\mathrm{mL} / \mathrm{min})$ & $10.7 \pm 3.8$ & $9.5 \pm 3$ & $12.1 \pm 4.3$ & 0.02 \\
\hline Maximal $\mathrm{O} 2$ pulse (\% PV) & $96 \pm 23$ & $82 \pm 18$ & $114 \pm 18$ & $<0.01$ \\
\hline $\mathrm{O} 2$ pulse $\geq 85 \% \mathrm{PV}$ & $29(57 \%)$ & $8(28 \%)$ & $21(95 \%)$ & $<0.01$ \\
\hline Wasserman stroke volume at exercise $(\mathrm{mL})$ & $72 \pm 29$ & $60 \pm 25$ & $85 \pm 28$ & 0.01 \\
\hline VE/VCO2 slope & $31 \pm 7$ & $32 \pm 18$ & $30 \pm 6$ & 0.55 \\
\hline \multicolumn{5}{|l|}{ Origin of altered peak VO2: } \\
\hline Cardiac limitation (\%) & & $20(69 \%)$ & & \\
\hline Respiratory limitation (\%) & & $1(3 \%)$ & & \\
\hline Muscular limitation (\%) & & $8(28 \%)$ & & \\
\hline
\end{tabular}

Data presented are number of patients (percent) or mean \pm SD. p-Value $<0.05$ was considered significant.

PV, predicted value; RER, respiratory exchange ratio; VE, ventilation; VCO2, carbon dioxide output. 
Table 4

Relationship between peak VO2 and clinical, biological or echocardiographic parameters at rest.

\begin{tabular}{|c|c|c|}
\hline Variables & $r^{2}$ & $\mathrm{p}$ \\
\hline \multicolumn{3}{|l|}{ Clinical } \\
\hline Age & 0.41 & $<0.01$ \\
\hline Female gender & 0.12 & 0.01 \\
\hline BMI $\left(\mathrm{kg} / \mathrm{m}^{2}\right)$ & 0.26 & $<0.01$ \\
\hline Diabetes mellitus & 0.18 & $<0.01$ \\
\hline Hypertension & 0.27 & $<0.01$ \\
\hline \multicolumn{3}{|l|}{ Biologic } \\
\hline Hemoglobin $(\mathrm{g} / \mathrm{dL})$ & 0.12 & 0.03 \\
\hline Log NT-proBNP (ng/mL) & 0.13 & 0.03 \\
\hline \multicolumn{3}{|l|}{ Echographic data at rest } \\
\hline Bicuspid valve & 0.14 & $<0.01$ \\
\hline Left atrial area $\left(\mathrm{cm}^{2}\right)$ & 0.1 & 0.02 \\
\hline $\mathrm{E} / \mathrm{e}^{\prime}$ ratio & 0.17 & $<0.01$ \\
\hline LV end-diastolic volume (mL) & 0.01 & 0.98 \\
\hline LV ejection fraction (\%) & 0.01 & 0.82 \\
\hline Global strain (\%) & 0.03 & 0.26 \\
\hline LV aortic valve area $\left(\mathrm{cm}^{2}\right)$ & 0.09 & 0.03 \\
\hline Aortic jet velocity (m/s) & 0.04 & 0.18 \\
\hline Aortic jet velocity $>4 \mathrm{~m} / \mathrm{s}$ & 0.01 & 0.91 \\
\hline Aortic jet velocity $>5 \mathrm{~m} / \mathrm{s}$ & 0.04 & 0.17 \\
\hline Mean transaortic pressure gradient & 0.02 & 0.27 \\
\hline LV stroke volume $(\mathrm{mL})$ & 0.01 & 0.75 \\
\hline Transtricuspid pressure gradient (mm Hg) & 0.15 & $<0.01$ \\
\hline TAPSE $(\mathrm{mm})$ & 0.04 & 0.17 \\
\hline
\end{tabular}

p-Value $<0.05$ was considered significant

BMI, Body Mass Index; LV, left ventricle; TAPSE, tricuspid annular plane systolic excursion.

responses to exercise are present in $\mathrm{HF}$ as a result of overactivity of ergoreceptors which are intramuscular afferents sensitive to products of skeletal muscle work [27]. All these conditions leading to peripheral deconditioning can explain the differences in exercise tolerance for similar level of AS severity. Peak VO2 provides perspective on the overall physiology of the body during maximal exercise but also during physical stress such as acute heart failure, systemic infection, and surgical stress. It is thus not surprising that peak VO2 is strongly linked to clinical outcome in our population as in other cardiovascular and noncardiovascular diseases [28]. Further studies should be performed to elucidate the exact role and determinants of peripheral (mal)adaptive mechanisms in this specific population.

We provide the first comprehensive exploration of AS patients with supine exercise echocardiography together with CPX. Dalsgaard et al. already demonstrated that peak exercise $\mathrm{E} / \mathrm{e}^{\prime}$ ratio was poorly correlated with invasive measurements of pulmonary capillary wedge pressure at rest in severe AS [29]. Accordingly, peak E/e' ratio was not

Table 5

Relationship between peak VO2 and exercise echocardiographic parameters.

\begin{tabular}{lll}
\hline Variables & $\mathrm{r}^{2}$ & $\mathrm{p}$ \\
\hline Echocardiographic data at exercise & & \\
E/e' ratio & 0.16 & 0.01 \\
LV ejection fraction (\%) & 0.01 & 0.52 \\
Global strain 25 W (\%) & 0.05 & 0.37 \\
LV aortic valve area $\left(\mathrm{cm}^{2}\right.$ ) & 0.09 & 0.03 \\
Aortic jet velocity $(\mathrm{m} / \mathrm{s})$ & 0.04 & 0.18 \\
Mean transaortic pressure gradient & 0.06 & 0.09 \\
LV stroke volume (mL) & 0.05 & 0.14 \\
Transtricuspid pressure gradient (mm Hg) & 0.01 & 0.75 \\
TAPSE (mm) & $<0.01$ & 0.7 \\
Exercise criteria of poor prognosis: & & \\
LV ejection fraction decrease $>3 \%$ & 0.01 & 0.87 \\
Mean gradient increase $>18 \mathrm{~mm}$ Hg & 0.01 & 0.60 \\
Exercise PHT $>$ 60 mm Hg & $<0.01$ & 0.52
\end{tabular}

p-Value $<0.05$ was considered significant

LV, left ventricle; PHT, pulmonary hypertension; TAPSE, tricuspid annular plane systolic excursion.
Table 6

Multiple regression analysis for prediction of peak VO2 in the whole population.

\begin{tabular}{llll}
\hline & Coefficient & Standard error & p value \\
\hline Age $($ per year $)$ & -0.18 & 0.03 & $<0.001$ \\
BMI $\left(\mathrm{kg} / \mathrm{m}^{2}\right)$ & -0.36 & 0.16 & 0.03 \\
Female gender & -4.9 & 1.3 & $<0.001$ \\
\hline
\end{tabular}

Potential predictors for peak VO2 with p-value $<0.05$ on bivariate analysis (age, BMI, hemoglobin, log NT-proBNP, left atrial area, E/E' moy, LV aortic valve area, transtricuspid pressure gradient, gender female, diabetes mellitus, hypertension, bicuspid valve) were added to the multivariate stepwise linear regression model using backward variable selection. Relevant variables were forced into the model (exercise PHT $>60 \mathrm{~mm} \mathrm{Hg}$, mean aortic gradient increase $>18 \mathrm{~mm} \mathrm{Hg}$, LV ejection fraction decrease $>3 \%$ ) because of their clinical relevance.

BMI, Body Mass Index.
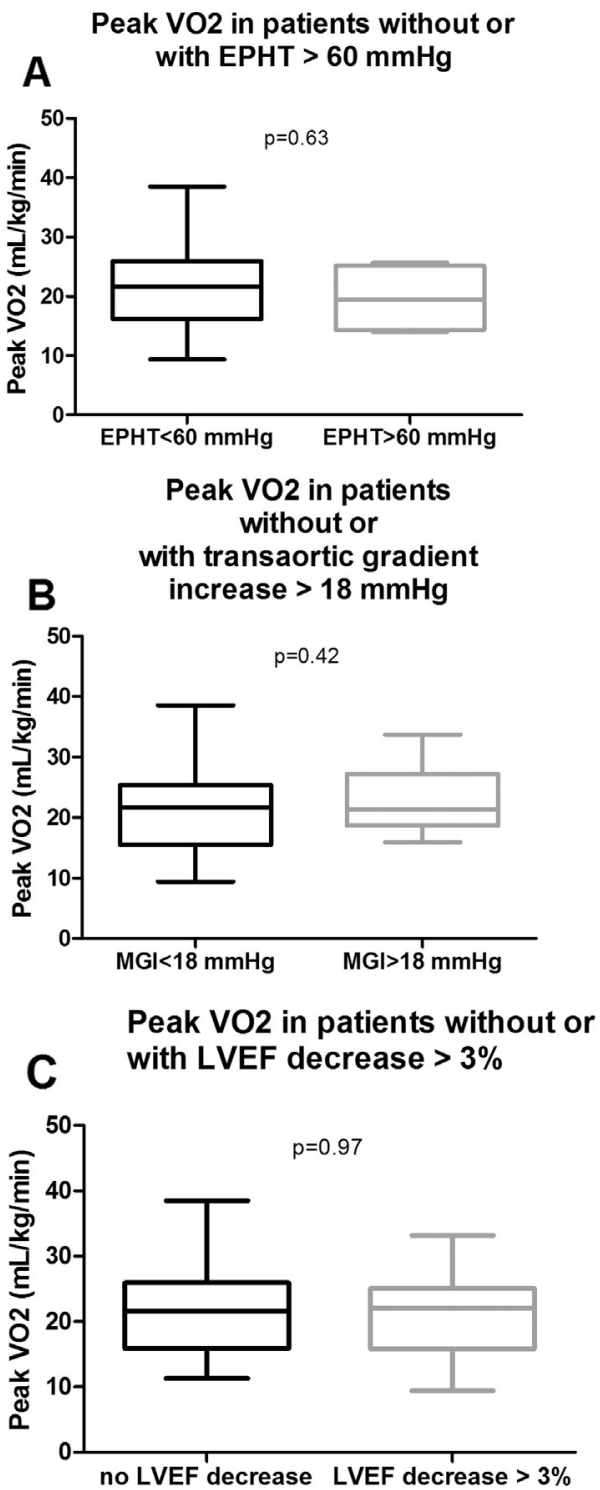

Fig. 1. Peak VO2 in patients with aortic stenosis grouped according to exercise echocardiographic severity criteria. Data are presented as mean \pm Standard Deviation, p-value was calculated using a Mann-Whitney U test. A. Patients with and without exercise pulmonary hypertension; B. patients with and without mean gradient increase $>18 \mathrm{~mm} \mathrm{Hg}$; C. patients with and without LVEF decrease. EPHT, exercise pulmonary hypertension; LVEF, left ventricle ejection fraction; MGI, mean gradient increase. 
A

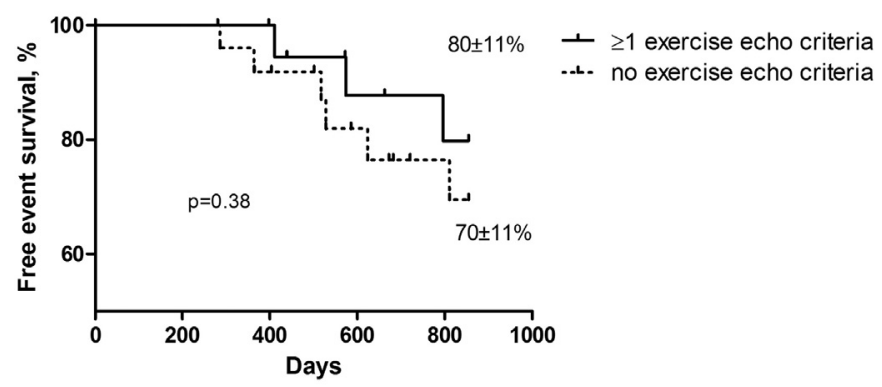

B

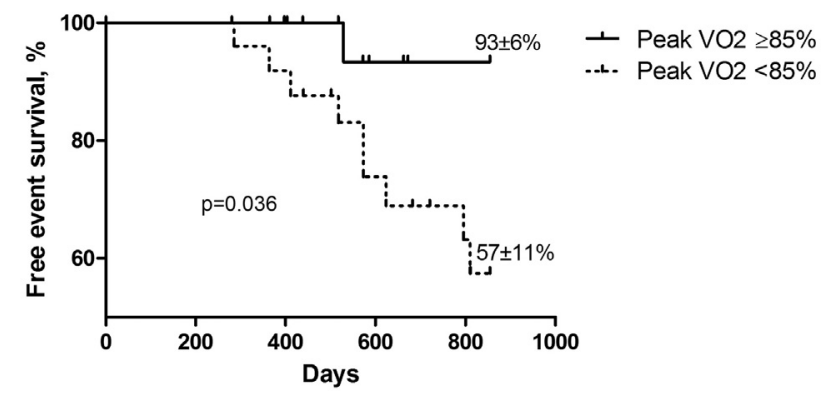

Fig. 2. Clinical outcome in asymptomatic patients with aortic stenosis. Kaplan-Meier curves according to: a. $\geq 1$ exercise criteria of bad prognosis (i.e. exercise PHT $>60 \mathrm{~mm} \mathrm{Hg}$, mean transaortic gradient increase $>18 \mathrm{~mm} \mathrm{Hg}$ and/or LVEF decrease $>3 \%$ ) (solid line) or no exercise criterion (dotted line) and b. peak $\mathrm{VO} 2 \geq 85 \%$ of predicted value (solid line) or peak $\mathrm{VO} 2<85 \%$ of predicted value (dotted line) at 28 months or 854 days follow-up. Events were death or cardiac surgery for symptom onset or alteration in LVEF. Note the significant excess event rate in patients with peak VO2 $<85 \%$ of predicted value. p-Value by log-rank test.

independently correlated with peak VO2 in our population. Surprisingly, we did not find any correlation between classical exercise echocardiographic parameters of asymptomatic AS severity (i.e. exercise PHT $>60 \mathrm{~mm} \mathrm{Hg}$, increase of mean transaortic gradient $>18 \mathrm{~mm} \mathrm{Hg}$ and decrease of $\mathrm{LVEF}>3 \%$ ) with peak VO2, suggesting that this parameter reflects more a general body adaptation and is thus well correlated with symptoms and outcomes.

\subsection{Risk stratification in asymptomatic AS}

We were able to demonstrate that altered peak VO2 $(<85 \%$ of predicted value) predicted poor outcome, with a lower event-free survival than preserved peak VO2 ( $\geq 85 \%$ of predicted value). In patients with severe LV dysfunction, peak VO2 is a well-proven determinant of survival, even in the setting of beta-blockade [30,31], so that CPX has a key role for heart transplantation selection in this population. Few recent studies highlighted its potential role in surgical selection of asymptomatic AS patients [12,13,23,24,32]. Levy et al. already proved in a small population of 43 asymptomatic AS patients that peak VO2 $\leq 14 \mathrm{~mL} / \mathrm{kg} / \mathrm{min}$ was associated with the occurrence of European Society of Cardiology guideline surgical class I triggers [12].

In the past few years, increase in mean transaortic pressure gradient $\geq 18$ to $20 \mathrm{~mm} \mathrm{Hg}$ [9,11], absence of LV contractile reserve [33], and exercise pulmonary hypertension $>60 \mathrm{~mm} \mathrm{Hg}$ [10] detected by supine exercise echocardiography were shown to have prognostic significance. Surprisingly, we were not able demonstrate any impact on survival of these criteria. Nevertheless our population was quite different, particularly younger, than those reported in these other studies, i.e. $54 \pm 21$ years old to be compared with $71 \pm 9$ years old in Lancellotti's study and $64 \pm 15$ years old in Marechaux's work. Thus our population had few cardiovascular risk factors, a high incidence of bicuspid valve (69\%), and a low rate of coronary heart disease. This was translated into the low cardiac event-rate (39\% at $21 \pm 7$ months) we observed in comparison to others.

\subsection{Clinical implications}

CPX may have a pivotal role in screening false asymptomatic AS patients in the future, as it objectively identifies low aerobic capacity in almost two thirds of patients, and thus patients with bad prognosis, who may be at higher risk of developing symptoms or LV dysfunction. Interestingly, normal aerobic capacity, i.e. VO2 peak $\geq 85 \%$ of predicted value, has an excellent negative predictive value of $97 \%$, when abnormal exercise testing guided by symptoms has a reported negative predictive value of 79 to $87 \%[34,35]$. CPX may thus be a very helpful tool within a watchful waiting strategy, and cannot be replaced by exercise echocardiography.

\section{Limitations}

Coronary arteriography was not realized in the whole population, so we cannot exclude that cardiac limitation was linked to coronary disease in some cases. However, our population was relatively young, with a large majority of bicuspid valves, and a low cardiovascular risk profile.

Our results are limited to 51 patients. However, all patients were carefully and prospectively underwent the three comprehensive exams (CPX, echocardiography at rest and exercise, biology) at the same period.

Aortic stenosis severity was evaluated in multiple transducer positions at rest as recommended, but only in the 5-chamber view during exercise because of patient's position, as previously reported by others [8]. This is an intrinsic limitation of aortic stenosis exploration during supine exercise which may result in underestimation of peak velocity. Yet the transaortic gradient increase was calculated with transaortic gradients obtained in 5 chamber views at rest and peak exercise.

\section{Conclusion}

In severe asymptomatic aortic stenosis patients, CPX objectively detects a high proportion of false "asymptomatic" patients secondary to cardiac limitation. Decreased aerobic capacity cannot be predicted by supine exercise echocardiography. Conserved aerobic capacity (peak VO2 $\geq 85 \%$ of predicted value) identifies a low risk population, for which watchful waiting strategy appears relevant. Conversely, false asymptomatic patients (defined by peak VO2 $<85 \%$ of predicted value) are faced with significant cardiac events and should be referred to the surgeon.

\section{Perspectives}

As elective surgery cannot be recommended in every patient with asymptomatic severe aortic stenosis, reliable risk estimation and appropriate aortic valve replacement indication and timing are important clinical challenges. Cardiopulmonary exercise testing detects a high proportion of false asymptomatic aortic stenosis patients with low peak VO2 associated with poor outcome that is not predicted by resting or supine exercise echocardiographic parameters of aortic stenosis severity. Since peak VO2 $\geq 85 \%$ of predicted value revealed to have an excellent negative predictive value, assessment of aerobic capacity using peak oxygen consumption should be part of current approaches to guide physician's decision making within a "watchful waiting" strategy.

\section{Conflict of interest}

The authors report no relationships that could be construed as a conflict of interest. 


\section{References}

[1] Joint Task Force on the Management of Valvular Heart Disease of the European Society of Cardiology (ESC), European Association for Cardio-Thoracic Surgery (EACTS), A. Vahanian, O. Alfieri, Andreotti, et al., Guidelines on the management of valvular heart disease (version 2012), Eur. Heart J. 33 (2012) 2451-2496.

[2] D.H. Kang, S.J. Park, J.H. Rim, et al., Early surgery versus conventional treatment in asymptomatic very severe aortic stenosis, Circulation 121 (2010) 1502-1509.

[3] T. Taniguchi, T. Morimoto, H. Shiomi, et al., Initial surgical versus conservative strategies in patients with asymptomatic severe aortic stenosis, J. Am. Coll. Cardiol. 66 (2015) 2827-2838.

[4] D.H. Kang, J.Y. Jang, S.J. Park, et al., Watchful observation versus early aortic valve replacement for symptomatic patients with normal flow, low-gradient severe aortic stenosis, Heart 101 (2015) 1375-1381.

[5] J.A. Castillo-Moreno, I.A. García-Escribano, Might Outcome of Patients With Asymptomatic Severe AS Be Improved by an Initial Surgical Strategy? J. Am. Coll. Cardiol. 26 (67) (2016) 1971-1972.

[6] M.L. Brown, P.A. Pellikka, H.V. Schaff, et al., The benefits of early valve replacement in asymptomatic patients with severe aortic stenosis, J. Thorac. Cardiovasc. Surg. 135 (2008) 308-315.

[7] T. Le Tourneau, P.A. Pellikka, M.L. Brown, et al., Clinical outcome of asymptomatic severe aortic stenosis with medical and surgical management: importance of STS score at diagnosis, Ann. Thorac. Surg. 90 (2010) 1876-1883.

[8] P. Lancellotti, D. Karsera, G. Tumminello, et al., Determinants of an abnorma response to exercise in patients with asymptomatic valvular aortic stenosis, Eur. J. Echocardiogr. 9 (2008) 338-343.

[9] S. Maréchaux, Z. Hachicha, A. Bellouin, et al., Usefulness of exercise-stress echocardiography for risk stratification of true asymptomatic patients with aortic valve stenosis, Eur. Heart J. 31 (2010) 1390-1397.

[10] P. Lancellotti, J. Magne, E. Donal, et al., Determinants and prognostic significance of exercise pulmonary hypertension in asymptomatic severe aortic stenosis, Circulation 126 (2012) 851-859.

[11] P. Lancellotti, F. Lebois, M. Simon, et al., Prognostic importance of quantitative exercise Doppler echocardiography in asymptomatic valvular aortic stenosis, Circulation 112 (2005) I377-I382.

[12] F. Levy, N. Fayad, A. Jeu, et al., The value of cardiopulmonary exercise testing in individuals with apparently asymptomatic severe aortic stenosis: a pilot study, Arch. Cardiovasc. Dis. 107 (2014) 519-528.

[13] A. Dhoble, M. Enriquez-Sarano, S.L. Kopecky, et al., Cardiopulmonary responses to exercise and its utility in patients with aortic stenosis, Am. J. Cardiol. 113 (2014) $1711-1716$.

[14] R.A. Nishimura, C.M. Otto, R.O. Bonow, et al., American College of Cardiology/American Heart Association Task Force on Practice Guidelines. 2014 AHA/ACC guideline for the management of patients with valvular heart disease: executive summary: a report of the American College of Cardiology/American Heart Association Task Force on Practice Guidelines, J. Am. Coll. Cardiol. 63 (2014) 2438-2488.

[15] H. Baumgartner, J. Hung, J. Bermejo, et al., American Society of Echocardiography; European Association of Echocardiography. Echocardiographic assessment of valve stenosis: EAE/ASE recommendations for clinical practice, J. Am. Soc. Echocardiogr. 22 (2009) 1-23.

[16] S.F. Nagueh, C.P. Appleton, T.C. Gillebert, et al., Recommendations for the evaluation of left ventricular diastolic function by echocardiography, Eur. J. Echocardiogr. 10 (2009) 165-193.

[17] C.M. Otto, A.S. Pearlman, K.A. Comess, R.P. Reamer, C.L. Janko, L.L. Huntsman, Determination of the stenotic aortic valve area in adults using Doppler echocardiography, J. Am. Coll. Cardiol. 7 (1986) 509-517.
[18] Z. Hachicha, J.G. Dumesnil, P. Bogaty, P. Pibarot, Paradoxical low-flow, low-gradient severe aortic stenosis despite preserved ejection fraction is associated with higher afterload and reduced survival, Circulation 115 (2007) 2856-2864.

[19] A. Coisne, F. Levy, D. Malaquin, et al., Feasibility of Doppler hemodynamic evaluation of primary and secondary mitral regurgitation during exercise echocardiography, Int. J. Card. Imaging 31 (2015) 291-299.

[20] R. Neviere, D. Montaigne, L. Benhamed, et al., Cardiopulmonary response following surgical repair of pectus excavatum in adult patients, Eur. J. Cardiothorac. Surg. 40 (2011) 77-82.

[21] K. Wasserman, J.E. Hansen, et al., Principles of Exercise Testing, Lippincott Williams \& Wilkins, 2005 71-102.

[22] D. Messika-Zeitoun, B.D. Johnson, V. Nkomo, et al., Cardiopulmonary exercise testing determination of functional capacity in mitral regurgitation: physiologic and outcome implications, J. Am. Coll. Cardiol. 47 (2006) 2521-2527.

[23] R. Dulgheru, J. Magne, L. Davin, et al., Left ventricular regional function and maximal exercise capacity in aortic stenosis, Eur. Heart J. Cardiovasc. Imaging 17 (2016) 217-224.

[24] R. Dulgheru, P. Pibarot, P. Lancellotti, et al., Impact of global hemodynamic load on exercise capacity in aortic stenosis, Int. J. Cardiol. 168 (2013) 2272-2277.

[25] C.D. Steadman, M. Jerosch-Herold, B. Grundy, et al., Determinants and functional significance of myocardial perfusion reserve in severe aortic stenosis, JACC Cardiovasc. Imaging 5 (2012) 182-189.

[26] M.J. Haykowsky, C.R. Tomczak, J.M. Scott, D.I. Paterson, D.W. Kitzman, Determinants of exercise tolerance in patients with heart failure and reduced or preserved ejection fraction, J. Appl. Physiol. 119 (1985) 739-744 (2015).

[27] A.C. Scott, et al., Chemical mediators of the muscle ergoreflex in chronic heart failure: a putative role for prostaglandins in reflex ventilatory control, Circulation 106 (2002) 214-220.

[28] M. Guazzi, et al. EACPR/AHA Scientific Statement Clinical recommendations for cardiopulmonary exercise testing data assessment in specific patient populations, Circulation 126 (2012) 2261-2274.

[29] M. Dalsgaard, J. Kjaergaard, R. Pecini, et al., Left ventricular filling pressure estimation at rest and during exercise in patients with severe aortic valve stenosis: comparison of echocardiographic and invasive measurements, J. Am. Soc. Echocardiogr. 22 (2009) 343-349.

[30] D.M. Mancini. H. Eisen, W. Kussmaul, et al, Value of peak exercise oxygen consumption for optimal timing of cardiac transplantation in ambulatory patients with heart failure, Circulation 83 (1991) 778-786.

[31] J.O. O'Neill, J.B. Young, C.E. Pothier, M.S. Lauer, Peak oxygen consumption as a predictor of death in patients with heart failure receiving beta-blockers, Circulation 111 (2005) 2313-2318.

[32] A. Dominguez-Rodriguez, P. Abreu-Gonzalez, C. Mendez-Vargas, et al., Ventilatory efficiency predicts adverse cardiovascular events in asymptomatic patients with severe aortic stenosis and preserved ejection fraction, Int. J. Cardiol. 177 (2014) $1116-1118$.

[33] P.V. Ennezat, J. Toussaint, S. Maréchaux, et al., Low-level exercise echocardiography fails to elicit left ventricular contractile reserve in patients with aortic stenosis, reduced ejection fraction, and low transvalvular gradient, Echocardiography 24 (2007) 47-51.

[34] P. Das, H. Rimington, J. Chambers, Exercise testing to stratify risk in aortic stenosis, Eur. Heart J. 26 (2005) 1309-1313.

[35] A.M. Rafique, S. Biner, I. Ray, J.S. Forrester, K. Tolstrup, R.J. Siegel, Meta-analysis of prognostic value of stress testing in patients with asymptomatic severe aortic stenosis, Am. J. Cardiol. 104 (2009) 972-977. 